\title{
Effets des facteurs endogènes et exogènes sur la croissance de la crevette géante d'eau douce Macrobranchium rosenbergii De Man, 1879 (Decapoda : Palaemonidae) le long du fleuve Wouri au Cameroun
}

\author{
Catherine Nicole DOUME DOUME ${ }^{1 *}$, Aboubacar TOGUYENI ${ }^{2}$ et Stanislas Silvain YAO $^{3}$ \\ ${ }^{1}$ Département de Pêche Industrielle, Faculté de Génie Industriel, Université de Douala, BP 2701 Douala, \\ Cameroun. \\ ${ }^{2}$ Laboratoire d'Etudes des Ressources Naturelles et des Sciences de l'Environnement (LERNSE), Institut du \\ Développement Rural, Université Polytechnique de Bobo - Dioulasso (UPB), 01 BP 1091 Bobo - Dioulasso \\ 01, Burkina Faso. \\ ${ }^{3}$ Laboratoire d'Hydrobiologie, UFR Biosciences, Université de Cocody-Abidjan, 22 B.P. 582 Abidjan 22, \\ Côte d'Ivoire. \\ *Auteur correspondant, E-mail :cathydoume@yahoo.fr ; Tél : +237-99-96-42-87.
}

\section{RESUME}

Cette étude a pour objectif d'estimer les paramètres de croissance de 432 échantillons de Macrobranchium rosenbergii (De Man, 1879) collectés le long du fleuve Wouri entre les mois de juillet 2010 et janvier 2012 à l'aide de filets éperviers et de nasses appâtées, puis transportés au laboratoire. Chaque spécimen a été mesuré, pesé et sexé. L'étude fournit des informations sur le sex-ratio, la relation longueur totale (LT) - longueur céphalothoracique (LC); LT - W (poids) et le facteur de condition K. La LT des individus capturés était comprise entre 66,0 à $111,0 \mathrm{~mm}$ avec les valeurs correspondantes de la LC de 23,16 à $39,28 \mathrm{~mm}$. Les poids corporels individuels ont varié de 6,15 à 8,40 g. Les résultats obtenus montrent que le sex-ratio est significativement en faveur des femelles et varie avec la taille. Les relations LT - LC et LT - W ont été hautement significatives ( $\mathrm{p}<0,0001$ ) dans les différents sites. Cette étude préliminaire de la croissance de $M$. rosenbergii le long du fleuve Wouri montre que cette espèce a une croissance isométrique et un facteur de condition $\mathrm{K}$ supérieur à 1 (moyenne $=1,066 \pm 0,261$ ). Elle pourrait donc être exploitée dans cette zone comme espèce potentielle d'aquaculture.

(C) 2013 International Formulae Group. All rights reserved.

Mots clés: Sex ratio, longueur totale, longueur céphalothoracique, poids, facteur de condition, isométrie.

\section{INTRODUCTION}

Macrobranchium rosenbergii (De Man, 1879) ou crevette, ou bouquet géant d'eau douce, appartient à l'ordre des décapodes Natantia, du sous-ordre des Caridés et de la famille des Palaemonidés. Originaire de l'Inde, elle a été introduite pour l'élevage en Amérique du Sud et en Afrique de l'Ouest. La production mondiale de Macrobranchium rosenbergii est de 229417 tonnes (FAO, 2009). Malgré des potentialités reconnues, l'Afrique reste le grand absent du développement crevetticole mondial (FAO, 2010). Au Cameroun, en raison de l'absence 
de toute forme d'élevage de crustacés, la production totale des crevettes provient exclusivement de la pêche. Cette exploitation intensive et non contrôlée pourrait entraîner une raréfaction voir un épuisement de cette ressource, ce qui provoquera la baisse de gains économiques des filières de pêche dépendantes et par conséquent la dégradation des conditions sociales des populations tributaires de cette ressource riche en protéines d'origine animale. Il est donc important, voire primordial de procéder à sa mise en captivité. Jusqu'à présent, aucune étude sur l'estimation des paramètres de croissance pondérale et linéaire n'a été réalisée sur cette espèce au Cameroun. L'objectif de la présente étude est d'estimer la répartition quantitative et spatio-temporelle de même que les paramètres de croissance des populations de Macrobranchium rosenbergii dans trois localités situées le long du fleuve Wouri (Bonassama, Manoka, Djébalé).

\section{MATERIEL ET METHODES}

Zones de l'étude et sites de collecte des crevettes

\section{Zone de l'étude}

La région du Littoral est située dans la partie méridionale de la plaine côtière. Elle s'étend entre le $2^{\mathrm{e}}$ et le $5^{\mathrm{e}}$ degré de latitude Nord à l'endroit où l'Océan Atlantique pénètre le plus profondément dans les terres. Elle occupe une superficie de $20248 \mathrm{~km}^{2}$. La région est divisée en quatre départements: Wouri, Sanaga-Maritime, Nkam, Moungo (wikipedia.org). Le fleuve Wouri, pièce maîtresse de l'Estuaire du Cameroun, a un débit de $308 \mathrm{~m}^{3} / \mathrm{s}$ et draine de nombreux sédiments à la mer. Le fond est constitué d'une vase molle noirâtre recouverte de débris végétaux. La salinité des eaux sur le fond est inférieure à $20 \%$ et la température varie de 25 à $33,5^{\circ} \mathrm{C}$ selon les saisons. Cette région et ses environs sont soumis à un climat équatorial de type camerounéen. La pluviométrie moyenne annuelle est de $3600 \mathrm{~mm}$ et les températures moyennes varient entre $24,7-25,2^{\circ}$ et 25,8 $27,6{ }^{\circ} \mathrm{C}$. Le réseau hydrographique est très dense. Il comprend plusieurs grands fleuves dont le Wouri, le Mungo, la Sanaga, qui communiquent entre eux à travers des mangroves marécageuses. Ces trois fleuves se jettent dans la mer par une large zone d'estuaires. La faune aquatique exploitable des eaux lagunaire et maritime est essentiellement constituée de poissons, de mollusques et de crustacés (Tiotsop, 2005). Pour cette étude, les sites de collecte de crevettes choisis (proximité des pêcheries, zones de frayères ou de migration des crevettes, accès facile) sont situés dans l'embouchure du fleuve Wouri (Figure 1), très riche en crevettes. Deux lots d'échantillons ont été prélevés à Bonassama $\left(4^{\circ} 04^{\prime} 28.92\right.$ ' $\mathrm{N}$ - 941'07.91'’E) et Djébalé (407'48.46' 'N $\left.9^{\circ} 43^{\prime} 31.67^{\prime} \mathrm{E}\right)$, tous deux situés dans un chenal de mangrove se jetant dans le fleuve et le troisième dans la baie de Manoka (349'04.82'’ N - 940'49.40'’E).

\section{Collectes des données}

Les crevettes vivantes, sont récoltées mensuellement entre les mois de juillet 2010 et janvier 2012 à l'aide de filets éperviers et de nasses appâtées. Elles sont ensuite transportées dans des sacs plastiques remplis d'oxygène au laboratoire. Au total, 432 individus de Macrobranchium rosenbergii ont été collectés: 193 au niveau de Bonassama, 94 à Djébalé et 145 à Manoka. Chaque spécimen a été mesuré et pesé. Les différents paramètres biométriques ont été mesurés à l'aide d'un pied à coulisse (lecture à $0,02 \mathrm{~mm}$ près), d'une balance électronique (Sartorius Compétence, précision 0,01 g). Le sexage est effectué d'après les critères énoncés par Pérez-Farfante et modifiés par Ismaël et New (2000).

\section{Caractéristiques morphologiques}

Les principaux paramètres mesurés sont :

- La longueur totale (LT), qui va de la pointe du rostre à celle du telson toujours sur la ligne médiodorsale ;

- La longueur céphalothoracique (LC), qui va de la marge postérieure du creux orbital au bord postérieur du céphalothorax 


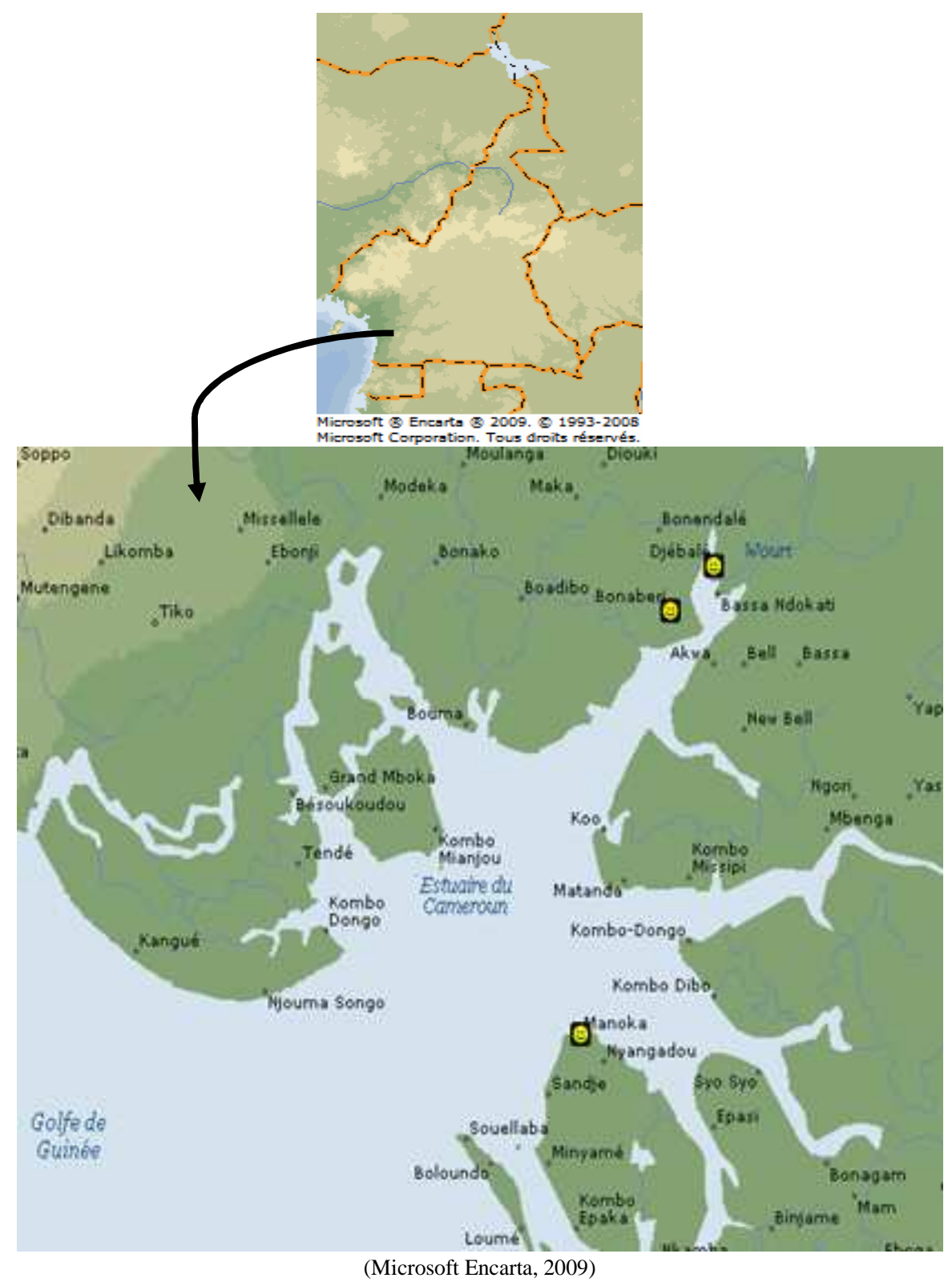

Figure 1: Embouchure du fleuve Wouri et sites de collecte des crevettes.

- $\quad$ Les relations LT/LC, LT/W et LC/W sont déterminées par une régression linéaire selon la méthode des moindres carrées. Les équations sont les suivantes :

$\mathrm{LT}=\mathrm{a}+\mathrm{b} \mathrm{LC} \quad$ et $\mathrm{W}=\mathrm{a} \times \mathrm{LT}^{\mathrm{b}}$ où $\mathrm{W}$ est le poids à l'état frais en gramme, LT la longueur totale en centimètre, LC la longueur céphalothoracique et $\mathrm{a}, \mathrm{b}$ sont les paramètres à déterminer, a étant une constante de régression ou ordonné à l'origine et b le coefficient d'allométrie. Ces paramètres de la relation taille-poids ont été calculés par sexe. Cette relation peut être linéarisée par transformation logarithmique de l'expression précédente afin de réduire la variabilité et d'homogénéiser les deux variables (W et LT) : $\ln \mathrm{W}=\ln \mathrm{a}+\mathrm{b} \ln \mathrm{Lf}$ avec $\ln =$ logarithme népérien. 
- $\quad$ Le facteur de condition $\mathrm{K}$

Il est calculé suivant Ricker (1975) : K = 100 $\mathrm{x} \mathrm{W} / \mathrm{LT}^{\mathrm{b}}$; $\mathrm{K}$ est calculé en fonction du mois, du sexe, du site, et de la taille.

- Les crevettes sont regroupées par classe de taille de $10 \mathrm{~cm}$ d'intervalle. Les fréquences de distribution de la population sont établies en fonction du mois et de la taille des crevettes.

\section{Sex-ratio}

Il est défini comme étant le pourcentage des individus mâles ou femelles par rapport à l'effectif total. Il est calculé selon la formule de Kartas et Quignard (1984) :

$\mathrm{SR}=\mathrm{Nf} / \mathrm{Nf}+\mathrm{Nm} * 100$; Où Nf $=$ nombre des femelles et $\mathrm{Nm}=$ nombre des mâles.

\section{Analyses statistiques}

Les moyennes des mesures entre les sous-échantillons correspondant, d'une part, aux deux sexes et d'autre part, aux habitats et aux moments de capture ont été comparées avec le test ANOVA I. La signification statistique du coefficient de régression $\mathrm{r}^{2}$ a été estimée et le test $\mathrm{t}$ de Student appliqué pour vérifier si les valeurs de $b$ diffèrent significativement de 3 à l'aide de la formule suivante: $\mathrm{t}=\mathrm{b}-3 / \sqrt{ } \operatorname{var}(\mathrm{b})$ avec $\operatorname{var}(\mathrm{b})=$ $\mathrm{Se}^{2} /(\mathrm{n}-1) \mathrm{Sx}^{2}$. Toutes ces analyses ont été effectuées à l'aide du logiciel PAST version 2.04. Les graphiques sont réalisés à l'aide des logiciels Microsoft Office Excel 2010 et Sigma Plot 11.0.

\section{RESULTATS}

\section{Caractéristiques morphométriques et fréquences de distribution}

La longueur totale chez les 432 individus récoltés le long du fleuve Wouri varie entre 66,0 et $111,0 \mathrm{~mm}$ tandis que la longueur céphalothoracique est comprise entre 23,16 et $39,28 \mathrm{~mm}$. Le poids corporel individuel quant à lui varie de 6,15 à $8,40 \mathrm{~g}$. Les fréquences de distribution en fonction du nombre d'individus et de la taille sont représentées dans les Figures 2(a) et 2(b). La distribution mensuelle est uni modale: le mode le plus élevé se situant entre les mois de Septembre et Octobre (Figure 2).

\section{Sex - ratio}

La répartition des sexes chez Macrobranchium est donnée dans le Tableau 1. Globalement, les femelles sont plus nombreuses que les mâles $(65,34 \%$ de femelles).

\section{Relation longueur totale-longueur céphalothoracique}

Les différents graphiques révèlent une corrélation positive entre la longueur totale et la longueur céphalothoracique chez Macrobranchium rosenbergii le long du fleuve Wouri. Cette relation présente des coefficients de détermination proche de l'unité (Figure 3).

\section{Relation longueur totale - poids}

L'équation générale de la relation longueur totale - poids corporel le long du fleuve Wouri est : $\mathrm{W}=0,0084 \mathrm{LT}^{2,9079}$ avec un $r^{2}=0,9974$ (Figure 4a). On note une variation $d u$ coefficient $b$ qui passe d'une allométrie négative $(\mathrm{b}<3)$ dans les sites de Bonassama et Manoka à une croissance isométrique $(b=3,0001)$ dans le site de Djébalé. Nous constatons que les valeurs du paramètre $b$ restent voisines quelque soit le site et pour l'ensemble de la population. L'application de test statistique de $\mathrm{t}$ - Student (Tableau 4) sur les coefficients de régression b au seuil de $5 \%$ indique une croissance allométrique minorante $(\mathrm{t}=-2,56)$. Les coefficients de détermination sont hautement significatifs au niveau de tous les sites.

\section{Facteur de condition $\mathbf{K}$}

Les valeurs mensuelles du facteur de condition et les types de croissance sont présentés dans le Tableau 2. Il en ressort qu'elles varient de 0,850 à 1,304 (moyenne $=$ $1,066 \pm 0,261$ ). Les Figures $5 \mathrm{a}$ et $5 \mathrm{~b}$ illustrent les variations de $\mathrm{K}$ en fonction du site et de la classe de taille et révèlent qu'il est supérieur à 
1 chez les individus de petite taille (taille comprise entre 6 et $9 \mathrm{~cm}$ ) et inférieur à 1 pour les plus gros spécimens. Considérant le sexe, le facteur de condition a varié de 0,885 à 1,495 (moyenne $=1,061 \pm 0,41$ ) chez les femelles et de 0,885 à 1,318 chez les mâles (moyenne $=1,101 \pm 0,433$ ). Toutefois, aucune différence significative n'est observée entre les sexes $(\mathrm{P}<0,01)$. Les valeurs les plus élévées, 1,216 et 1,$318 ; 1,319$ et 1,495 ont été respectivement enregistrées en saison des pluies (Juillet, Août 2010) chez les mâles et en Novembre, décembre 2011 chez les femelles. Les résultats de l'analyse comparative entre les différents sites à travers une ANOVA I (K, site, mois, sexe) sont consignés dans le Tableau 3. Ces résultats montrent qu'il n'y a pas de différence significative entre les mâles et les femelles d'un même site mais aussi entre deux sites différents.
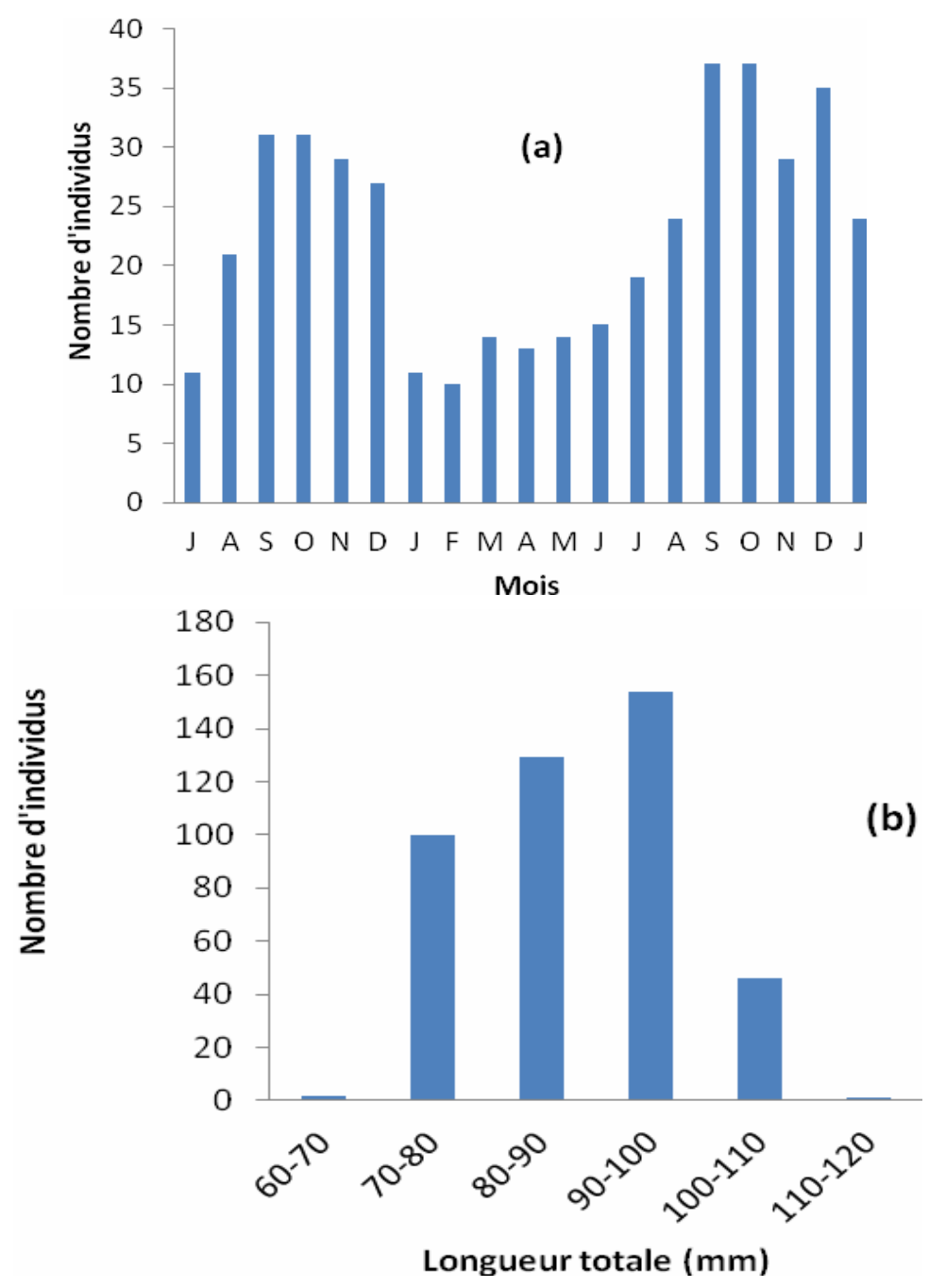

Figure 2: Fréquence de distribution : (a) mensuelle du nombre d'individus de juillet 2010 à janvier 2012 et (b) de la taille de Macrobranchium rosenbergii le long du fleuve Wouri. 


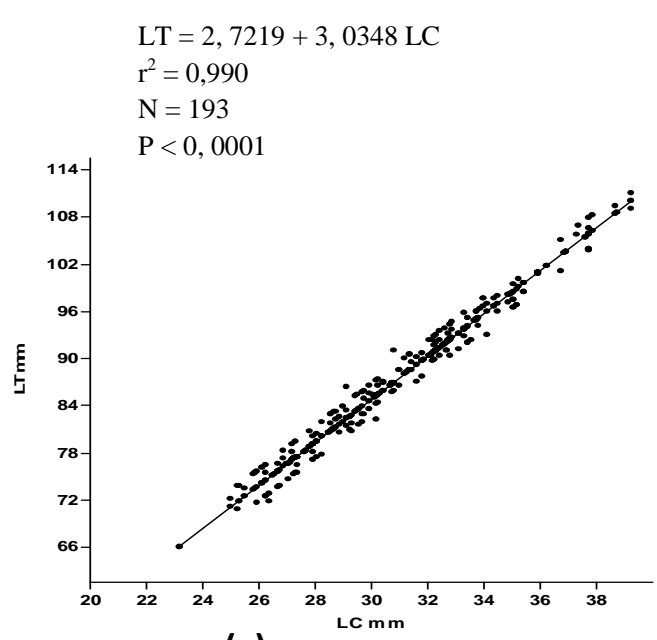

(a)

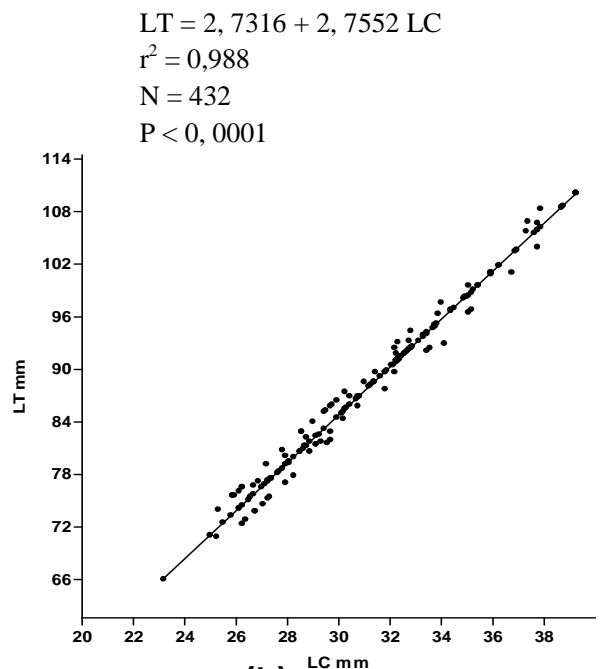

(b)
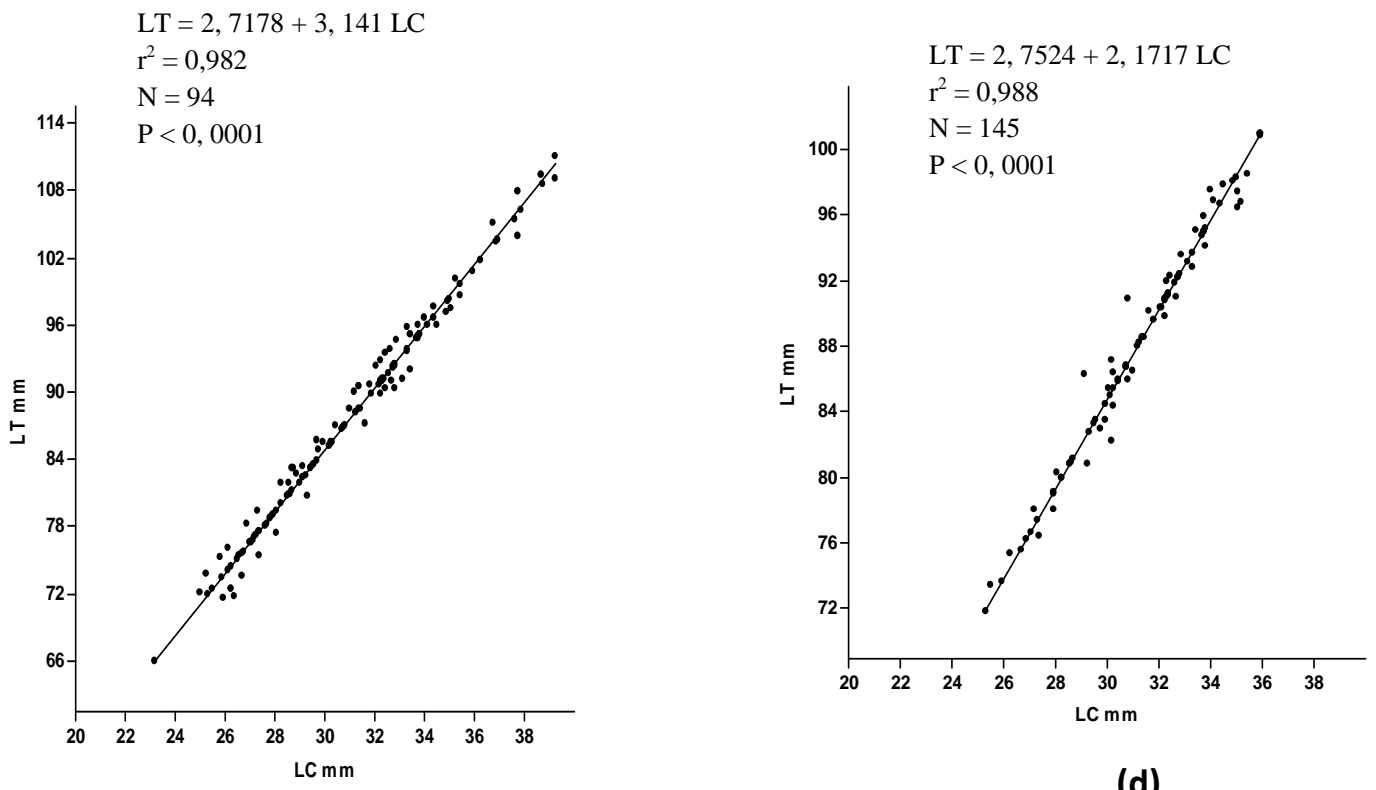

(c)

Figure 3: Relation longueur totale (LT) - longueur céphalothoracique (LC) chez Macrobranchium rosenbergii récoltées mensuellement le long du fleuve Wouri de juillet 2010 à janvier 2012. (a)Général ; (b) - Bonassama ; (c) - Manoka et (d) - Djébalé 

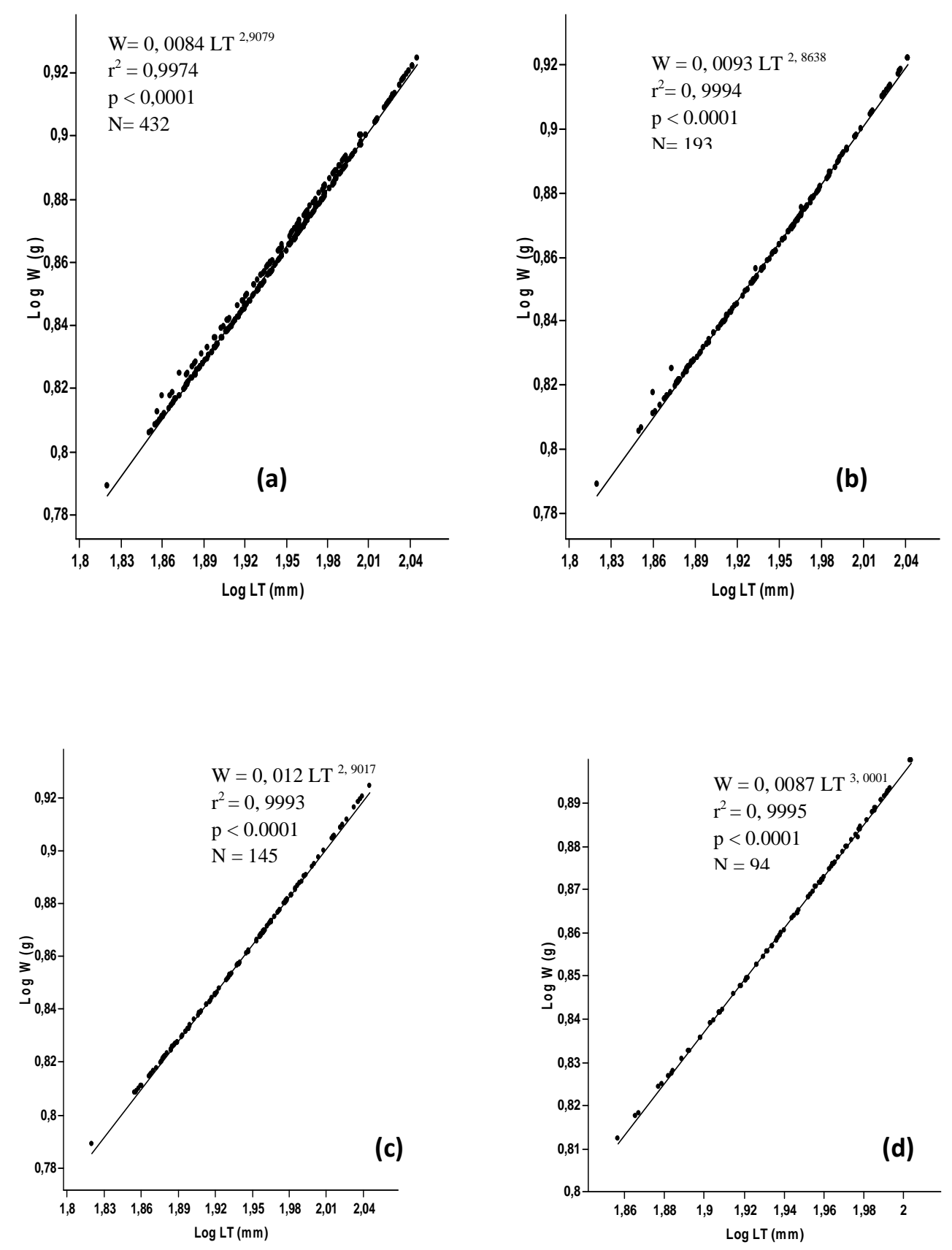

Figure 4: Relation entre la longueur totale (LT) et le poids (W) chez Macrobranchium rosenbergii le long du fleuve Wouri de juillet 2010 à janvier 2012. (a) - Général ; (b) - Bonassama ; (c) - Manoka; (d) - Djébalé 

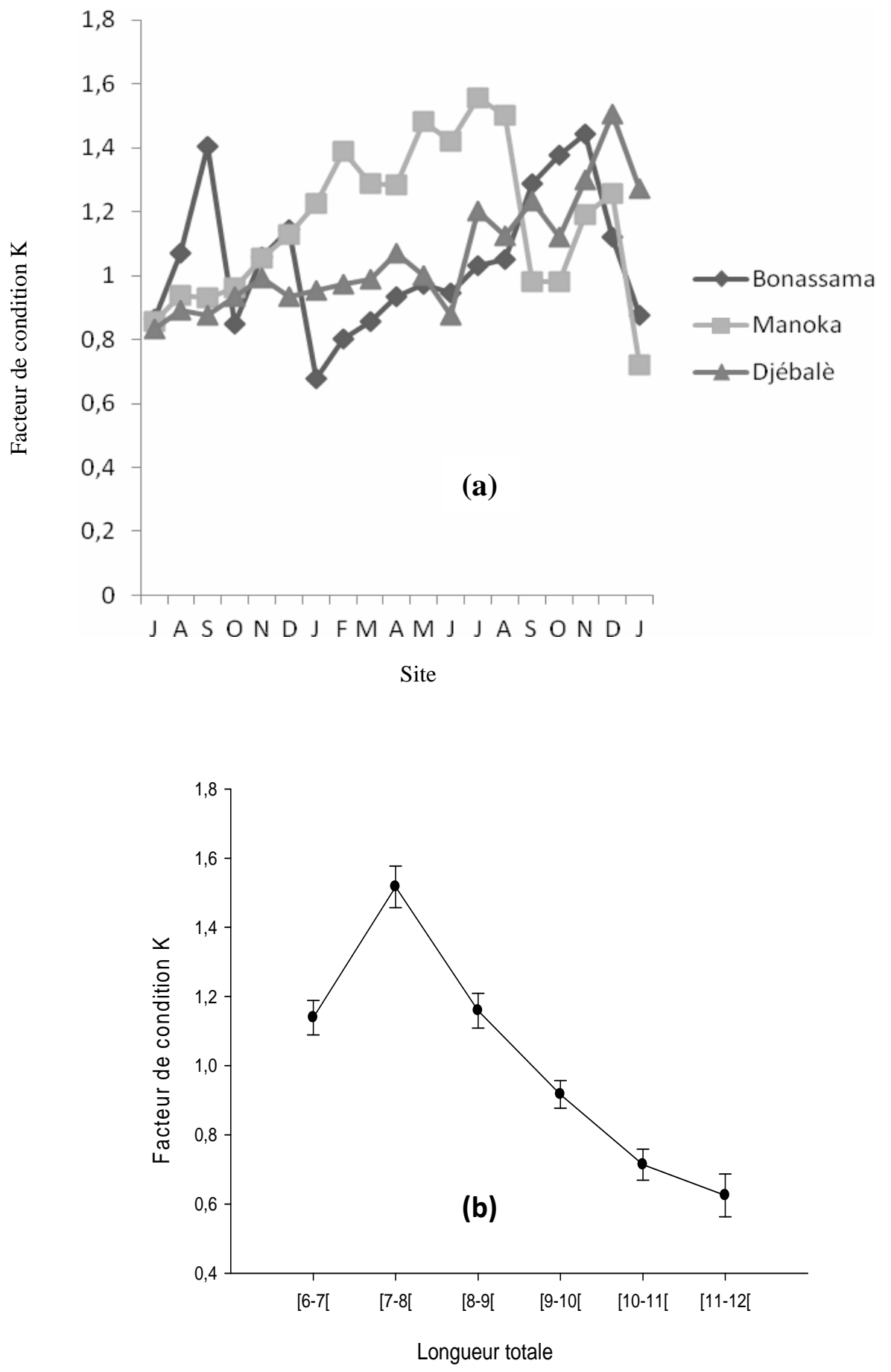

Figure 5: Evolution du facteur de condition $\mathrm{K}$ en fonction du site (a) et des classes de taille (b) chez Macrobranchium rosenbergii le long du fleuve Wouri (juillet 2010 - janvier 2012). 
Tableau 1: Répartition mensuelle du sex-ratio (\% de femelles) chez Macrobranchium rosenbergii en fonction des sites de capture le long du fleuve Wouri.

\begin{tabular}{lccccc}
\hline Périodes de capture & N & Bonassama & Manoka & Djébalé & Moyenne mensuelle \\
\hline Juil.2010 & 11 & 80 & 75 & 50 & 68,3 \\
Août & 21 & 71,4 & 77,8 & 60 & 69,7 \\
Sept & 31 & 75 & 71,4 & 66,7 & 71 \\
Oct. & 31 & 76,9 & 72,7 & 51,1 & 66,9 \\
Nov. & 29 & 66,7 & 71,4 & 75 & 71 \\
Déc. & 27 & 62,5 & 66,7 & 60 & 63 \\
Jan 2011 & 11 & 80 & 75 & 50 & 68,3 \\
Fév. & 10 & 80 & 66,7 & 50 & 65,6 \\
Mars & 14 & 75 & 50 & 75 & 66,7 \\
Avril & 13 & 66,7 & 50 & 66,7 & 61,1 \\
Mai & 14 & 71,4 & 50 & 66,7 & 62,7 \\
Juin & 15 & 66,7 & 75 & 50 & 63,9 \\
Juil. & 19 & 62,5 & 66,7 & 60 & 63 \\
Août & 24 & 77,8 & 60 & 60 & 65,9 \\
Sept & 37 & 76,9 & 75 & 75 & 75,6 \\
Oct. & 37 & 66,7 & 76,9 & 66,7 & 70,1 \\
Nov. & 29 & 81,3 & 75 & 80 & 78,8 \\
Déc. & 35 & 37,5 & 72,7 & 25 & 45,1 \\
Janv.2012 & 24 & 33,3 & 66,7 & 33,3 & 44,4 \\
Total & 432 & 68,86 & 68,14 & 59,01 & 65,32 \\
\hline \multicolumn{5}{r}{ N- Nombre d'échantillons par mois }
\end{tabular}

Tableau 2: Facteur de condition $\mathrm{K}$ et type de croissance chez Macrobranchium rosenbergii le long du fleuve Wouri (Juillet 2010 - Janvier 2012).

\begin{tabular}{lccc}
\hline Mois & $\mathbf{k}$ & Type de croissance & $\mathbf{N}$ \\
\hline Juil.2010 & $0,850 \pm 0,046$ & Allométrie négative & 11 \\
Août & $0,962 \pm 0,033$ & Allométrie négative & 21 \\
Sept & $1,033 \pm 0,025$ & Isométrique & 31 \\
Oct. & $0,915 \pm 0,030$ & Allométrie négative & 31 \\
Nov. & $1,032 \pm 0,042$ & Allométrie négative & 29 \\
Déc. & $1,059 \pm 0,117$ & Allométrie négative & 27 \\
Jan 2011 & $0,911 \pm 0,034$ & Allométrie négative & 11 \\
Fév. & $1,014 \pm 0,009$ & Allométrie négative & 10 \\
Mars & $1,022 \pm 0,020$ & Allométrie négative & 14 \\
Avril & $1,083 \pm 0,007$ & Allométrie négative & 13 \\
Mai & $1,12 \pm 0,054$ & Allométrie négative & 14 \\
Juin & $1,044 \pm 0,032$ & Allométrie négative & 15 \\
Juil. & $1,235 \pm 0,083$ & Isométrique & 19 \\
Août & $1,20 \pm 0,018$ & Isométrique & 24 \\
Sept & $1,15 \pm 0,040$ & Isométrique & 37 \\
Oct. & $1,144 \pm 0,040$ & Isométrique & 37 \\
Nov. & $1,304 \pm 0,015$ & Isométrique & 29 \\
Déc. & $1,257 \pm 0,237$ & Isométrique & 35 \\
Janv.2012 & $0,917 \pm 0,021$ & Isométrique & 24 \\
Total & $1,066 \pm 0,261$ & Isométrique & 432 \\
\hline & $\mathrm{K}=$ facteur de condition $\cdot \mathrm{N}=$ nombre de crevettes &
\end{tabular}


Tableau 3: Données de l'analyse de la variance sur le facteur de condition en relation avec le sexe, le lieu et la période de capture chez Macrobranchium rosenbergii le long du fleuve Wouri (Juillet 2010 - Janvier 2012).

\begin{tabular}{lccccccc}
\hline $\begin{array}{l}\text { Sources de } \\
\text { variations }\end{array}$ & Somme des carrés & $\begin{array}{c}\text { Degré de } \\
\text { liberté }\end{array}$ & $\begin{array}{c}\text { Carrés } \\
\text { moyens }\end{array}$ & $\mathbf{F}$ & $\mathbf{P}$ & $\mathbf{F}_{\mathbf{0}, \mathbf{0 5}}$ & $\mathbf{F}_{\mathbf{0}, \mathbf{0 1}}$ \\
\hline Intergroupes & 0,3744 & 7 & & & & & \\
intragroupes & 6,5866 & 144 & 0,0535 & 1,169 & 0,3 & 2,08 & 2,79 \\
Total & 6,9810 & 151 & 0,4574 & & & & \\
\hline
\end{tabular}

Tableau 4: Test $\mathrm{t}$ - Student.

\begin{tabular}{ccccccc}
\hline & $\mathbf{a}$ & $\mathbf{b}$ & $\mathbf{r}^{2}$ & $\mathbf{n}$ & $\sqrt{ } \mathbf{v a r}(\mathbf{b})$ & t-Student \\
\hline Général & 0,0084 & 2,9079 & 0,9974 & 432 & 0,036 & $-2,56$ \\
Bonassama & 0,0093 & 2,8638 & 0,9994 & 193 & 0,023 & $-5,92$ \\
Manoka & 0,012 & 2,9017 & 0,9993 & 145 & 0,018 & $-5,46$ \\
Djébalè & 0,0087 & 3,0001 & 0,9995 & 94 & 0,00004 & 2,5 \\
\hline
\end{tabular}

\section{DISCUSSION}

La taille maximale observée chez Macrobrachium rosenbergii le long du fleuve Wouri a été de 111,0 mm LT chez un mâle, correspondant à un poids de $8,40 \mathrm{~g}$. Des données similaires ont été signalées par Marioghae (1987) au Nigéria et Durand et al. (1994) en Côte d'Ivoire. Cette espèce appartient au genre Macrobranchium (Rüppel, 1836) qui comprend des espèces de tailles assez grandes à l'état adulte (Griessinger et al., 1990). Elle pourrait atteindre des tailles plus grandes (jusqu'à $30 \mathrm{~cm}$ pour $250 \mathrm{~g}$ ). Il existe un dimorphisme sexuel, chez cette espèce, les mâles étant généralement plus développés que les femelles. Selon Fontaine et al. (2009), cette caractéristique biologique est un bon indicateur des performances zootechniques susceptibles de s'exprimer en milieu d'élevage. En effet, la taille maximale d'un poisson est en général un indicateur de sa vitesse de croissance et les plus grandes espèces sont celles qui possèdent le meilleur potentiel d'adaptation à différents milieux (Legendre et Albaret, 1991). Ces résultats préliminaires sur la biologie de $M$. rosenbergii le long du fleuve Wouri montrent une fluctuation mensuelle aussi bien en nombre qu'en taille des individus. Les modes les plus élevés se situent en Septembre et en Octobre 2011 et montrent que $88,43 \%$ de la population ont une taille comprise entre 70 et $100 \mathrm{~mm}$ LT. Ceci pourrait être lié aux méthodes de pêche utilisées, aux types d'habitats échantillonnés, aux périodes d'échantillonnage, et à une disparition d'espèces (espérance de vie très courte) comme mentionné dans les travaux de Kouamélan et al. (2003). Il convient cependant de souligner que la distribution et l'abondance des macros invertébrés comme les crevettes dans les cours d'eau, est dans une large mesure, fonction des exigences spécifiques des divers taxa et des caractéristiques environnementales des différentes portions des rivières, ainsi que de la disponibilité de la nourriture et la nature du substrat (Troadec et al., 1969 ; Hynes, 1970 ). Ceci pourrait expliquer l'importance du nombre d'individus collectés en saison des pluies (Juillet, Août, Septembre, Octobre). Ces résultats viennent d'ailleurs corroborer 
ceux obtenus par Marioghae (1987, 1995) au Nigeria. Ces résultats suggèrent que la saison des pluies, qui entraine des modifications dans l'habitat, serait favorable à la croissance des crevettes. En effet, c'est pendant cette période que l'on observe une plus grande disponibilité de l'alimentation naturelle, notamment le phytoplancton et le zooplancton. Aussi, les jeunes crevettes pêchées sont les plus vulnérables aux engins de pêche liée au fait qu'en saison pluvieuse, elles sortent de leurs niches, attirées par la richesse en substances nutritives des eaux du fleuve. Pendant la saison sèche (Décembre, janvier, février, mars), la plupart des habitats s'assèchent et la collecte des échantillons devient difficile. L'analyse du sex-ratio révèle une différence significative $(p<0,0001)$ en faveur des femelles durant toute la saison des pluies (Nwosu, 2013). La dominance des femelles dans les captures pendant cette période tient au fait que ces espèces se reproduisent en saison des pluies (Goore Bi, 1998). Elles seraient donc plus vulnérables du fait de l'activité de reproduction. Ainsi, la variation dans l'abondance des femelles dans les captures serait le reflet du déroulement du cycle de reproduction de l'espèce comme souligné par Demestre (1990). Concernant, les relations taille-poids, les valeurs du coefficient d'allométrie $b$ diffèrent de 3 dans les deux sexes ce qui suggère que $M$. rosenbergii ne suit pas strictement la loi du cube. Ce coefficient donne en effet des informations sur le type de croissance relative $\mathrm{du}$ poids et de la longueur des poissons (Montcho et al., 2009); La croissance de $M$. rosenbergii du fleuve Wouri a varié dans l'ensemble de l'allométrie négative $(b=2,65)$ à l'isométrie $(\mathrm{b}=3,00)$. Ces résultats sont concordants avec ceux habituellement rapportés dans la littérature et qui situeraient la valeur du coefficient b entre 2,50 et 3,50 (Pauly et Gayanilo, 1997) ou entre 2,50 et 4,00 (Offem et al., 2009) ou encore entre 2,00 et 4,00 (Montchowui et al., 2009). Des différences sont observées entre le site de Djebalé où la croissance est isométrique et les sites de Bonassama et Manoka dans lesquels les individus ont eu une croissance en faveur de la longueur (allométrie négative). Ces tendances sont contraires à celles observées dans la basse vallée de l'Ouémé au cours des travaux de Kouton (2004) et de Wénon (2005). Ces déviations pourraient s'expliquer par le fait que $b$ dépendrait tant des facteurs biotiques que abiotiques, et surtout de la disponibilité des aliments et du type de l'habitat (Arslan et al., 2004). Toutefois, la valeur élevée de $r^{2}(0,9974)$ révèle chez cette espèce, une étroite corrélation entre le poids corporel et la longueur totale. Des résultats analogues ont été obtenus chez d'autres espèces de crevettes caridées de l'Afrique de l'Ouest: Macrobranchium macrobranchion $\left(\mathrm{r}^{2}=0,968\right)$ et Nematopalaemon hastatus Aurivillius $\left(\mathrm{r}^{2}=0,895\right)$ provenant de la rivière Cross au Nigeria (Enin, 1994 ; Nwosu et al., 2006). L'analyse du facteur de condition $K$ n'a pas révélé de différences significatives entre les mâles et les femelles. Toutefois, des différences sont observées entre les sites, celui de Manoka ayant la plus grande valeur. Le facteur de condition étant un paramètre révélateur de l'état de condition du poisson et donc un indicateur relatif des interactions entre les facteurs biotiques et abiotiques sur les conditions physiologiques (Lizana et al., 2002). La valeur élevée de $\mathrm{K}$ dans ce site pourrait s'expliquer par la richesse de cette zone qui draine de grandes quantités de sédiments. D'après Fulton (1902), une valeur de $K \geq 1$ exprimerait un état de bien-être d'une population au cours des stades variés de son cycle de vie, alors que $K<1$ signifierait que l'animal n'est pas en embonpoint dans son biotope. Le bien-être de la population de $M$. rosenbergii se serait donc bien exprimé dans l'écosystème du fleuve Wouri au cours de cette période d'étude. En effet, la première 
phase qui correspond à la valeur faible et stationnaire de $\mathrm{K}$ coïncide avec la période de préparation à la maturation et à la période de maturation des gonades de $M$. rosenbergii (Hattour, 2000). La valeur faible de K peut être expliquée par le fait que les réserves sont investies dans l'élaboration des produits sexuels (Lalèyè et al., 1995). Après la période de ponte, qui s'achève en Juillet - août, $M$. rosenbergii se nourrit abondamment tout en accumulant des réserves qui se traduisent par une augmentation de $\mathrm{K}$ aux mois de septembre, octobre, novembre et décembre. En effet, cette variation de $\mathrm{K}$ est due principalement au comportement alimentaire de l'espèce et à la disponibilité de l'alimentation (El Oumari et al., 2011).

\section{Conclusion}

$\mathrm{Au}$ terme de cette étude sur la croissance de la crevette géante d'eau douce, Macrobranchium rosenbergii, le long du fleuve Wouri en fonction des facteurs endogènes et exogènes, il ressort que c'est une espèce de grande taille qui a une croissance isométrique et un facteur de condition $\mathrm{K}$ supérieur à 1 dans la zone. Elle pourrait donc être exploitée comme espèce potentielle d'aquaculture, afin de lutter contre le déficit protéinique constaté dans la zone. De plus, cette étude a permis de comprendre la biologie de cette espèce assez mal connue en Afrique centrale.

\section{REMERCIEMENTS :}

Les auteurs remercient particulièrement l'équipe de l'écloserie - AQUABIOCAM qui a gracieusement mis ses locaux à sa disposition pour les analyses des échantillons ; ainsi que tous les pêcheurs qui ont contribué à l'identification des sites de capture des crevettes, et à la collecte de données biologiques.

\section{REFERENCES}

Arslan M, Ayhan Y, Serdar B. 2004. Length - weight relationships of Brown Trout, Salma trutta L., Inhabiting Kan Stream, Coruh Basin, North - Eastern Turkey. Turkish J. Fisheries and Aquat. Sci., 4: 45-48.

Demestre M, Fortuno JM. 1992. Reproduction of the deep-water shrimp Aristeus antennatus (Decapoda: Dendrobranchiata), Barcelona, Spain. Marine Ecology Progress Series, 84: 4151.

Durand JR, Loubens G. 1994. Biologie et dynamique des populations d'Alestes baremoze (Pisces, Characidae) du bassin de l'Ayamé, Côte d'Ivoire. Travaux et Documents de l'ORSTOM, 98: 332 .

Enin UL. 1994. Length-weight parameters and condition $\mathrm{k}$ factor of two West African prawns (1). Rev. Hydrobiology. Frop., 27(2): 121-127.

Enin UL. 1995. First estimates of growth, mortality and recruitment parameters of Macrobachium macrobrachion (Herklots, 1851) in the Cross River Estuary, Nigeria. Dana, 11(1): 29-38.

El Oumari N, Mohammed M, Hachem I. 2011. Etude de la croissance de Pagellus acarne: le cas de la côte Méditerranéenne marocaine. J. Sci. Hal. Aquat., 4: 110119.

FAO. 2009. La situation mondiale des pêches et de l'aquaculture, FAO.

FAO. 2010. Méthodes simples pour l'aquaculture. Collection FAO Formation. http://apps.fao.org/page/collections?subse $\mathrm{t}=$ nutrition consulté le 10-12-2012.

Fontaine $\mathrm{P}$, Legendre $\mathrm{M}$, Vandeputte $\mathrm{M}$, Fostier A. 2009. Domestication de nouvelles espèces et développement durable de la pisciculture. Cah. Agric., 18(2-3): 119-124. 
Fulton. 1902. Rate of growth of seas fishes. Sci. Invest. Fish. Div. Scot. Rept., 20: 1035-1039.

Goore Bi G. 1998. Contribution à l'étude des crevettes d'eau douce de la Côte d'Ivoire: systématique, biologie et analyse socioéconomique de la pêche de Macrobrachium vollenhovenii (Herklots, 1857) et Macrobrachium macrobrachion (Herklots, 1851) (Crustacea : 64 Decapoda, Palaemonidae) du bassin de la Bia. Thèse de doctorat du $3^{\text {ème }}$ cycle, Université de Cocody, République de la Côte d'Ivoire, 143 p.

Griessinger JM, Lacroix D, Gondouin P. 1991. L'Elevage de la Crevette Tropicale d'Eau Douce. Ifremer ; 372 p.

Hattour A. 2000. Contribution à l'étude des poissons pélagiques des eaux tunisiennes. Thèse de Doctorat, Faculté des Sciences de Tunis, l'Institut National des Sciences et Technologies de la Mer, Tunis, 212 p.

Hynes HB. 1970. The Ecology of Running Waters. University of Toronto Press: Toronto.

Ismael D, New MB. 2000. Freshwater Prawn Culture: the Farming of Macrobrachium rosenbergii. Blackwell Science: Oxford, England; 18-40.

Kartas F, Quignard JP. 1984. Contribution à l'étude de l'allache (Sardinella aurita Val. 1847) des côtes de Libye. Rapp. Comm. Int. Mer Médit., 23(8): 157- 176.

Kouamélan EP, Teugels GG, N'Douba V, Goore Bi G, Kone T. 2003. Fish diversity and its relationships with environmental variables in a West African basin. Hydrobiologia, 505: 139-146.

Kouton MD. 2004. Diversité, écologie et exploitation des crevettes d'eau douce dans la basse vallée de l'Ouémé : cas de la commune d'adjohoun au Bénin. Thèse d'ingénieur Agronome, FSA/UAC, 100 p.

Lalèyè P, Baras E, Philippart JC. 1995. Variations du régime alimentaire de
Chrysichthys nigrodigitatus et $C$. auratus (Claroteidae) dans les lagunes du SudBénin. Aquatic Living Resources, 8: 365372.

Legendre M, Albaret JJ. 1991. Maximum observed length (MOL) as an indicator of growth rate in tropical fishes. Aquaculture, 94: 327 - 341.

Lizana MD, Ambrosio AM. 2002. Condition factor of nine species of fish of the Characidae family in the upper Parana River floodplain, Brazil. Braz. J. Biol., 62(1): 113-124.

Marioghae IE. 1987. Notes on the biology and distribution of Macrobrachium vollenhovenii and Macrobachium macrobrachion in the Lagos Lagoon (Crustacea, decapoda,palemonidae). Rev. Zool. Af., 94(3): 493-508.

Marioghae IE. 1995. Review of research on penaed shrimp in Nigeria, in the Mangrove Ecosystem in the Niger Delta region of Nigeria. Proceeding of a workshop organized at the University of Port-Harcourt, 212-225.

Montcho SA, Lalèyè $P$, Linsenmair EK. 2009. Length-length, length-weight relationships and condition factor of Nile perch late Niloticus (Linnaeus, 1762) in the Pendjari River, West Africa. Int. J. Biol. Chim. Sci., 3(3): 468-474.

Montchowui E, Niyonkuru C, Lalèyè $\mathrm{P}$, Ahouansou-Montcho S, Chikou A. 2009. Weight-length relationship for commercial fish species caught in Lake Hlan in Benin (West Africa). Int. J. Biol. Chem. Sci., 3(3): 612-616.

Nwosu FM, Wolfi M. 2006. Population dynamics of the giant African river prawn Macrobrachium vollenhovenii, Herklots 1857 (Crustacea, Palaemonidae) in the Cross River estuary, Nigeria. West African Journal of Applied Ecology, 9: 118. 
Nwosu FM. 2013. Management Implications of Sex Ratios of three Palaemonid Shrimps in the Cross River Estuary, South-East, Nigeria. Journal of Fisheries and Aquatic Science, 8(1): 291-294.

Offem BO, Samsons YA, Omoniyi IT. 2009. Length-weight relationship, Condition factor and sex Ratio of Forty six Important Fishes in a Tropical Flood River. Research Journal of Fisheries and Hydrobiology, 4(2): 65-72.

Pauly D, Gayanilo FC. 1997. An alternative approach to estimating the parameters of length-weight relationship from length frequency samples and their bulk weights. The FAO-ICLARM Stock Assessment Tool (FiSAT). Reference Manual FAO Comp. Inf. Ser. Fish. 8, ICLARM, Manila, Philippines, 262 p.

Ricker WE. 1980. Calcul et interprétation des statistiques biologiques des populations de poissons. Bull. office des recherches sur les pêcheries du Canada 191F. Ottawa, 409 p.

Tiotsop F. 2005. Importance de la mangrove dans le système halieutique au Cameroun. Mémoire présenté à l'Université du Québec à Rimouski,. Université du Québec, 98 p.

Troadec JP. 1982. Introduction à l'aménagement des pêcheries: intérêt, difficultés, et principales méthodes. FAO Doc. Tech. Pêches, 224: 64 p.

Wénon D. 2005. Ecologie et exploitation des crevettes d'eau douce dans la basse vallée de l'Ouémé : cas d'Agonlin - lowé dans la commune d'Adjohoun. Mémoire pour l'obtention du Diplôme d'Ingénieur des Travaux (DIT), EPAC/UAC. 92 p. 